\title{
Efficient, Authenticated, and Fault-Tolerant Key Agreement for Dynamic Peer Groups
}

\author{
Li Zhou and Chinya V. Ravishankar
}

\author{
Department of Computer Science \& Engineering \\ University of California, Riverside \\ Riverside, CA 92521, USA \\ \{lzhou, ravi\}@cs.ucr.edu
}

\begin{abstract}
We present an efficient authenticated and fault-tolerant protocol $(A F T D)$ for tree-based key agreement. Our approach is driven by the insight that when a Diffie-Hellman blinded key is updated, in a tree-based method, it suffices to send the update to a small subset of the group, instead of entire group, as current methods require. Our scheme distributes each updated public key to a relatively small subgroup, called its trust set, greatly improving performance. Moreover, we use a threshold secret sharing method to distribute the function of the trusted authority across trust sets, thereby guaranteeing key authentication, enhancing fault-tolerance, and protecting our protocol from impersonation attacks. Our performance analysis suggests that our scheme significantly reduces the communication overhead and storage requirement.
\end{abstract}

Keywords: Secure Group Communication, Key Agreement, Key Authentication

\section{Introduction}

As a result of the increased popularity of group-oriented applications, such as pay-TV, distributed interactive games, teleconferencing and chat rooms, there is a growing demand for security services to achieve secure group communication. A common method is to encrypt messages with a group key, so that entities outside the group cannot decode them. A satisfactory group communication system would possess the properties of group key security, forward secrecy, backward secrecy, and key authentication/integrity $[1,2,3]$. In this paper, we focus on Key authentication/integrity, which ensures that public keys of group members cannot be modified by adversaries. There are two approaches for generating such group keys: centralized key distribution and distributed key agreement. Centralized key distribution uses a dedicated key server, resulting in simpler protocols. However, centralized methods fail entirely once the server is compromised, so that the central key server makes a tempting target for adversaries. In addition, centralized key distribution is not suitable for dynamic peer groups, in which all nodes play the same function and role, thus it is unreasonable to make one the 
key server, placing all trust in it. In contrast, distributed key agreement requires each member to contribute a share to generate the group key, resulting in more complex protocols.

The group key is updated on every membership change for forward and backward secrecy, a method called group rekeying. To reduce the number of rekeying operations, Wong et al. [4] proposed a logical data structure called a key tree that reduces the rekeying overhead from $O(n)$ to $O(\log n)$, where $n$ is the group size. Based on this idea, Kim et al. proposed a tree-based key agreement protocol, TGDH [1], which is a combination of key tree and Diffie-Hellman key exchange to generate and maintain the group key.

Unfortunately, TGDH suffers from two drawbacks. As explained in Section 3, it remains prone to impersonation attacks, and uses more messages than necessary.

\subsection{Our Work}

In this paper, we propose a novel Authenticated, Fault-tolerant Tree-based Diffie-Hellman key agreement protocol, AFTD, based on two key ideas. First, as explained in Section 3, it is gross overkill to broadcast updated public keys to all group members for recomputing the group key when a node $n_{i}$ joins or leaves. It suffices to send each update to a much smaller subset of nodes in the tree, called its trust set $T S\left(n_{i}\right)$. Second, we achieve robust key authentication by distributing the function of trusted authority among the nodes in $T S\left(n_{i}\right)$, using a threshold cryptographic scheme. Any $k$ members of a node's trust set can serve as its public key certificate authority. Our performance analysis shows this scheme can reduces the communication overhead from $O\left(n^{2}\right)$ to $O(n \log n)$ for initialization, and from $O(n \log n)$ to $O(n)$ for rekeying. It also reduces the storage requirement for blinded keys from $O(n)$ to $O(\log n)$. This feature is particularly useful when a broadcast channel is unavailable.

The rest of this paper is organized as follows. We survey related work in Section 2. Section 3 motivates our work and defines our intrusion model. We present our solution in Section 4 and demonstrate performance analysis and comparison in Section 5. Finally we make a conclusion in Section 6.

\section{Related Work}

Key trees $[4,5]$ were first proposed for centralized key distribution, while Kim et al. [1] adapted it to a distributed key agreement protocol TGDH. Every group member create a key tree separately. Each leaf node is associated with a real group member, while each non-leaf node corresponds to a subgroup of group $G$, considered a virtual member. In Figure 3 , virtual member $V_{4}$ corresponds to the subgroup that contains two real group members $M_{3}$ and $M_{4}$.

In $T G D H$, every node on the key tree has a Diffie-Hellman key pair based on the prime $p$ and generator $\alpha$, used to generate the group key. Secret-public key pair $\left\{K_{M_{i}}, B K_{M_{i}}=\alpha^{K_{M_{i}}} \bmod p\right\}$ is for real member $M_{i}$, and $\left\{K_{V_{i}}, B K_{V_{i}}=\right.$ 
$\left.\alpha^{K_{V_{i}}} \bmod p\right\}$ is for virtual member $V_{i}$. Public key $B K_{M_{i}}$ is also called as blinded $k e y$. Consider a node $M_{v}$ whose left child is $M_{l v}$ and right child node is $M_{r v}$ (to simplify the description, we do not distinguish real members from virtual members here). $M_{i}$ 's secret key can be computed in the usual Diffie-Hellman fashion as $K_{M_{v}} \equiv\left(B K_{l v}\right)^{K_{r v}} \equiv\left(B K_{r v}\right)^{K_{l v}} \bmod p$.

With all blinded keys well-known, each group member can compute the secret keys of all nodes on its key path, comprising the nodes from the leaf node up to the root. The root node's secret key $K_{V_{0}}$ is known to all group members, and becomes the group key. In Figure 3, group member $M_{2}$ knows the key pairs of $M_{2}, V_{3}, V_{1}$ and $V_{0}$. $V_{0}$ 's secret key is the group key.

Steiner et al. [6,2] proposed a family of Group Diffie-Hellman $(G D H)$ protocols for dynamic peer groups. Based on them, Ateniese et al. [7] proposed a new multiparty authenticated key agreement protocol, which offers key authentication/integrity, key confirmation, and non-repudiation of group membership. However, some flaws in this protocol have been found by Pereira et al. [8].

Lee et al. $[9,10]$ have designed several tree-based distributed key agreement protocols, reducing the rekeying complexity by performing interval based rekeying. They also present an authenticated key agreement protocol. As the success of their scheme is partially based on a certificate authority, their protocol will encounter the same problems as centralized trust mechanisms.

In [11], Kong et al. provide robust and ubiquitous security support for mobile ad-hoc networks. In their scheme, they distribute the certificate authority functions through a threshold secret sharing mechanism, in which each entity holds a secret share, and multiple entities in a one-hop neighborhood jointly provide certificate services. Our distributed trust mechanism differs from theirs in two aspects. First, our goals are different. Second, the nodes that offer valid certificates are different [12].

\section{Motivation and Attack Model}

$T G D H[1]$ is a simple and efficient approach for the establishment of ephemeral keys for group sessions, however, it suffers from two significant drawbacks.

First, although TGDH uses authenticated channels, it still seems vulnerable to impersonation attacks. To provide authenticated blinded keys, TGDH suggests that every protocol message be signed by its sender using some strong public signature method such as DSA or RSA, and then verified by all receivers using the sender's public key. However, TGDH is a session key generation protocol, and does not address the long-term security of DSA or RSA keys. Since adversaries can compromise those keys in the long run, these keys must be refreshed periodically from a trusted source that is available online. Our approach is to define a trust set for each member $M_{i}$, and distribute the function of trusted authority across trust sets so that any $k$ members from this set can offer $M_{i}$ 's public key certificate, enhancing fault-tolerance. We distribute the function of trust authority in a manner similar to the scheme in [11], which is based on $(k, n)$-threshold secret sharing scheme. It is pointed out in [13] that scheme 
in [11] is not usable in a group with malicious members since it does not provide an important property known as verifiability. However, we focus on the correct and secure generation of group keys in the face of outsider attacks mounted by non-group members as in $[14,15]$. We do not address insider attacks mounted by malicious group members because they can always reveal their own private keys or the group key to non-group members, thus causing fraudulent membership events or compromising group communication. Thus our scheme is unaffected by the flaw pointed out in [13]. Detailed description of intrusion model appears in [12].

Second, TGDH involves excessive communication and storage overheads caused by broadcasting updated blinded keys. This problem becomes more serious if membership events are common. Storage requirement also becomes an important issue when resource limited devices, such as PDAs, are able to join the group as qualified group members. In our scheme, instead of broadcasting these updates, they are transmitted to a smaller subset of nodes (trust set), so that communication and storage overheads can be reduced significantly.

\section{Our Solution}

\subsection{Overview}

$T G D H$ [1] observes that a node $n_{1}$ in the group needs to know the blinded key of another node $n_{2}$ only if $n_{2}$ is on its co-path, defined as the set of siblings of each node in the key path of $n_{1}$. However, we offer the key insight that even more is true. In fact, an update of a blinded key need be sent only to a small subset of the group, instead of entire group. Because of the way Diffie-Hellman key exchanges are used in a key tree to generate the group key, the blinded key of any node $n_{i}$ is only needed by the leaf nodes of the subtree rooted at $n_{i}$ 's sibling. This group of nodes, which we call $n_{i}$ 's trust set, forms the basis for both improved efficiency as well as key authentication. We send each node's blinded keys only to its trust set. A node's trust set is also entrusted with the task of responding to requests for its public key, and provides key certificates using a threshold cryptographic scheme. This insight is missing from earlier work.

Key Management Phases. In our scheme, each group member construct a key independently. Each real group member $M_{i}$ has two key pairs: a DiffieHellman key pair, $\left\{K_{M_{i}}, B K_{M_{i}}=\alpha^{K_{M_{i}}} \bmod p\right\}$, which is used to generate the group key, and an RSA secret-public key pair, $\left\{D_{i}, E_{i}\right\}$, which is used to provide source authentication. Non-leaf nodes $V_{i}$ are virtual members, and have only a Diffie-Hellman key pair $\left\{K_{V_{i}}, B K_{V_{i}}=\alpha^{K_{V_{i}}} \bmod p\right\}$.

Group key management in our approach occurs in two phases: the initialization phase and the rekeying phase. Initialization is a one-time activity that distributes appropriate public-key certificates to trust sets. While such initialization may be done in many ways, we simplify our presentation by postulating that this function is performed by a trusted authority (TA), which subsequently goes offline. Offline here means the TA will not issue renewed public key certificates for existing group members during the process of group rekeying. New 


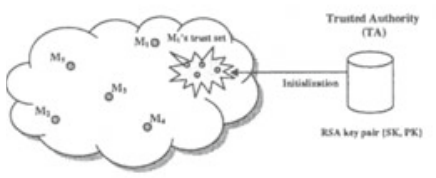

(a) Initialization Phase

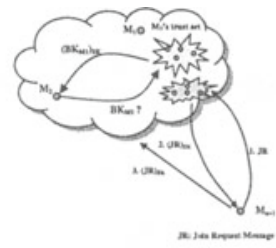

(b) Rekey Option 1: Certification by Peers

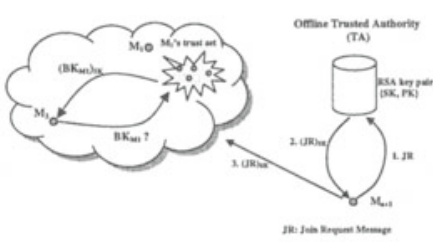

(c) Rekey Option 2: Certification by Offline Trusted Authority

Fig. 1. AFTD overview

members wishing to join the group may obtain initial certificate from the TA at any time prior to join.

This TA uses an RSA secret-public key pair $\{S K, P K\}$, and establishes public key certificates for each group member $M_{i}$ by signing $M_{i}$ 's public key with its secret key $S K . M_{i}$ 's public key certificate $\left\langle M_{i}, B K_{M_{i}}, E_{i}\right\rangle_{S K}$ is now distributed to its trust set. Since the public key $P K$ is well known, any member of $M_{i}$ 's trust set can verify this certificate and obtain $M_{i}$ 's public key.

The initialization phase also distributes a secret share $S K_{j}$ of the secret key $S K$ to each group member $M_{j}$ using Shamir's $(k, n)$-threshold sharing [16], which is used for creating partial public key certificates held by members of trust sets. A node $M_{j}$ in $M_{i}$ 's trust set verifies the original certificate for $M_{i}$ (signed using $S K$ ), and re-encrypts it with $S K_{j}$ to create a partial certificate. Now any $k$ members in the trust set of a given group member can offer that group member's public key certificate by group signing of certificates.

In the rekeying phase, AFTD includes protocols in support of three operations: join, leave and interval multicast.

\subsection{Initialization Phase}

Assume the group $G$ has $n$ real group members $M_{1}, M_{2}, \ldots, M_{n}$ initially. We describe how to distribute the function of the trusted authority to appropriate subgroups (trust set) so that any $k$ member nodes in an appropriate subgroup can offer the corresponding valid certificate. Here "valid" means the certificate has been signed with the system secret key $S K$.

Distributing the system secret key shares $S K_{i}$. Our design uses Shamir's $(k, n)$-threshold scheme [16]. First, the TA randomly selects a $(k-1)$ degree polynomial $f(x)=S K+a_{1} \cdot x+\cdots+a_{k-1} \cdot x^{k-1}$, such that the shared secret is $f(0)=S K$. Each group member obtains a secret share $S S_{M_{i}}=\left(f\left(M_{i}\right) \bmod m\right)$. For any $k$ group members $\left\{M_{1}, M_{2}, \ldots, M_{k}\right\}$, La- 
grange interpolation yields $S K \equiv \sum_{i=1}^{k}\left(S S_{M_{i}} \cdot l_{M_{i}}(0)\right) \equiv \sum_{i=1}^{k} S K_{i}(\bmod m)$, where $l_{M_{i}}(0)$ are the Lagrange coefficients ${ }^{1}$.

Obtaining valid certificates: The certificate $X$ for any node is served by the node's trust set, with each member in that trust set providing a partial certificate $X^{S K_{i}}$. With any $k$ partial certificates, the requesting member can compute the valid certificate as $X^{S K_{1}} \cdot X^{S K_{2}} \cdots X^{S K_{k}}=X^{\left(\sum_{i=1}^{k} S K_{i}\right)}=X^{S K}$ [11]. Thus, these $k$ members can work like a trusted authority, and jointly offer the certificate. (We use the t-bounded coalition offsetting algorithm proposed in [11] to ensure that the above equation is valid.)

This approach has the nice feature that the system secret key $S K$ is never revealed to any member node nor to any subset of member nodes. They can jointly reconstruct $X^{S K}$, but never $S K$ itself. While this method can be unsafe if group members can be compromised [13], this difficulty does not arise in our case, as explained in Section 3.

Further, AFTD improves fault-tolerance, since Shamir's threshold scheme ensures that any set of $k-1$ or less secret shares cannot jointly obtain $S K$. Thus if any set of $k-1$ or less secret shares have been discovered, the system secret key $S K$ is still safe from adversaries.

Defining Trust Sets. At the beginning, each group member is assigned a unique member ID and associated with a leaf node of the key tree in ascending order. To define trust sets, the group is first split into $k$-member clusters. The members in the last cluster may have more than $k$ group members when $n$ is not a multiple of $k$. The upper part of Figure 3 shows a 7-member group. When $k=2$, the group is divided into 3 clusters, and the last one has three members.

Definition 1 (Trust Set). The trust set of $M_{i}$ or $V_{i}$ (See Figure 2) is the set of nodes in the union of all clusters that contain one or more leaf nodes of the subtree rooted at $M_{i}$ or $V_{i}$ 's sibling node, and represented as $\operatorname{TS}\left(M_{i}\right)$ or $\operatorname{TS}\left(V_{i}\right)$.

In Figure 3, $T S\left(V_{2}\right)$ is the set of nodes in the first two clusters, which contain all leaf nodes $\left\{M_{1}, M_{2}, M_{3}, M_{4}\right\}$ of the subtree rooted at $V_{2}$ 's sibling node $V_{1}$.

When the number of clusters in $T S\left(M_{i}\right)$ is less than two, we improve fault tolerance by including in $T S\left(M_{i}\right)$ the first adjacent cluster formed by the leaves of the sibling of $M_{i}$.

Distributing the Certificates to Trust Sets. After distributing the system secret key shares to all group members, the trusted authority distributes the stored public key certificates to appropriate trust sets. Then the TA works offline and is used only to initialize new members joining the group. The system can provide key authentication service to renewed RSA keys without the help of the TA, since its function has been fully distributed to appropriate trust sets.

\subsection{The Rekeying Phase}

To achieve forward and backward secrecy (Section 1), the group key must be updated whenever new members join or old members leave. Each new member

${ }^{1}$ Defined as $l_{M_{i}}(x)=\frac{\left(x-M_{1}\right) \cdots\left(x-M_{i-1}\right)\left(x-M_{i+1}\right)\left(x-M_{k}\right)}{\left(M_{i}-M_{1}\right) \cdots\left(M_{i}-M_{i-1}\right)\left(M_{i}-M_{i+1}\right)\left(M_{i}-M_{k}\right)}$. 
$M_{J}$ must also obtain a system secret share $S S_{M_{J}}$ and some certificates so that it can offer certificate services.

Localized Self-initialization. When an uninitialized new member $M_{J}$ joins the group, a $k$-coalition of old members which are share holders $\left\{M_{J_{1}}, M_{J_{2}}, \cdots M_{J_{k}}\right\}$, can jointly offer a system secret share $S S_{M_{J}}$ to $M_{J}$ as follows:

$S S_{M_{J}} \equiv \sum_{i=1}^{k} S S_{M_{J_{i}}} \cdot l_{M_{J_{i}}}\left(M_{J}\right) \equiv \sum_{i=1}^{k} S S_{J_{i}}(\bmod m)$.

The Lagrange coefficients and $S S_{J_{i}}$ are known by $M_{J}$, so $S S_{M_{J_{1}}}$ can be derived directly. Here we use the shuffling scheme presented in [11] to maintain the secrecy of $M_{J_{i}}$ 's share.

The Join Protocol. Assume that a new member $M_{n+1}$ wishes to join a $n$-member group which contains $\left\{M_{1}, M_{2}, \ldots, M_{n}\right\} . M_{n+1}$ is required to authenticate itself by presenting a join request signed with $S K . M_{n+1}$ may obtain a signature on its join request either by establishing credentials with the offline trusted authority, or by enlisting the cooperation of at least $k$ nodes from the group $G$ willing to recognize and certify $M_{n+1}$ 's request.

When the other group members receive this request, they independently determine $M_{n+1}$ 's insertion node [1] in the key tree, which is the shallowest rightmost node, or the root node when the key tree is well-balanced. They also independently determine a real member called join sponsor $M_{S}[1]$ to take responsible for coordinating the join, which is the rightmost leaf node in the subtree rooted at the insertion node.

No keys change in the key tree at a join, except the blinded keys for nodes on the key path for the sponsor node. The sponsor simply recomputes the group key, and sends updates for blinded keys on its own key path to their corresponding trust sets. Each member $M_{j}$ of the sponsor node's trust set creates a new partial share $S S^{\prime}{ }_{j}$ of the secret key $S K$, and forwards it to $M_{n+1}$, which combines them to obtain its new secret share $S K_{n+1}$. The new node $M_{n+1}$ also sends its signed certificate to the members of its trust set $T S\left(M_{n+1}\right)$, and gets the public keys needed for generating the group key. The join works as shown in Algorithm 1.

\footnotetext{
Algorithm 1 Join Protocol in $A F T D$

1: The new member $M_{n+1}$ broadcasts the signed join request to the group.

2: Group members determine the insertion point, and update their key trees by creating a new intermediate node and promoting it to become the parent of both the insertion node and $M_{n+1}$.

3: Each group member adjusts the clusters in its key tree by adding $M_{n+1}$ to the smallest cluster adjacent to the insertion point, or to the cluster on its right one in case of a tie. If the size of the modified cluster goes up to $2 k$, split it into two clusters.

4: The sponsor $M_{S}$ computes the new group key, and sends the updated blinded keys of nodes on its key path to their corresponding trust sets. These messages are signed by the sponsor $M_{s}$.

5: The members in these trust sets request the sponsor $M_{S}$ 's certificate from $T S\left(M_{S}\right)$ to verify the updated blinded keys they received.

6: $M_{n+1}$ obtains its secret share $S K_{n+1}$ from $T S\left(M_{S}\right)$.

7: $M_{n+1}$ sends its valid public key certificates to its trust set, and gets the public keys needed for generating the group key.
}

In Figure 3, $M_{8}$ joins a 7 -member group, and $k=2$. The join sponsor $M_{7}$ creates a new intermediate node $V_{6}$ in the key tree and promotes it to become 


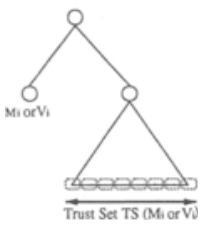

Fig. 2. Trust Set of $M_{i}$ or $V_{i}$

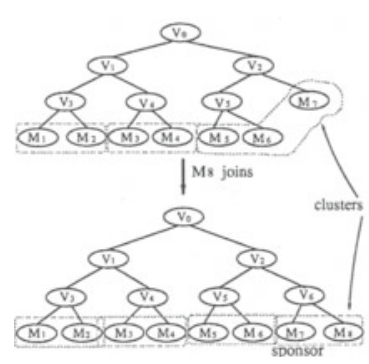

Fig. 3. Join Process in AFTD

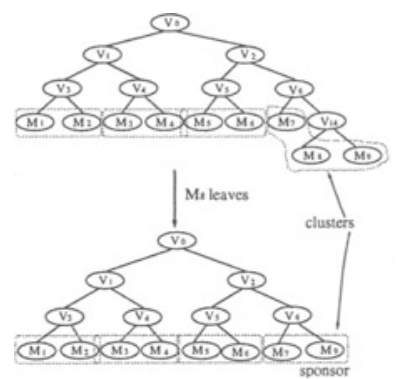

Fig. 4. Leave Process in AFTD

the parent of $M_{7}$ and $M_{8}$. The sponsor $M_{7}$ computes the new group key, sending the updated $B K_{V_{6}}$ and $B K_{V_{2}}$ to their corresponding trust sets $\left\{M_{5}, M_{6}, M_{7}, M_{8}\right\}$ and $\left\{M_{1}, M_{2}, M_{3}, M_{4}\right\}$ respectively. Finally as the size of the third cluster is extended to $2 k=4$, it splits into two clusters: $\left\{M_{5}, M_{6}\right\}$ and $\left\{M_{7}, M_{8}\right\}$.

Leave Protocol. Assume that a member $M_{L}$ wishes to leave a $n$-member group. First $M_{L}$ initiates the leave protocol by sending a leave request. When the other group members receive the request, they independently determine the sponsor node, which is defined as in [1] to be the right-most leaf node of the subtree rooted at the leaving member's sibling node. The leave protocol works as shown in Algorithm 2.

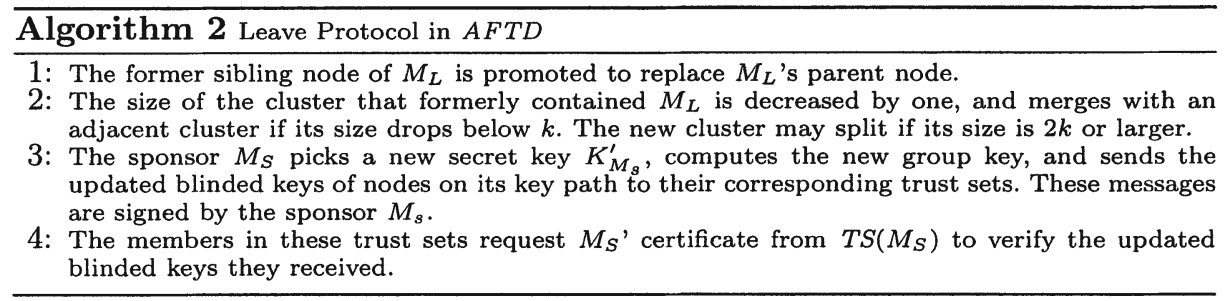

In Figure 4, $M_{8}$ leaves a 9 -member group where $k=2$. The sponsor $M_{9}$ picks a new secret key $K_{M_{9}}$ and computes the new group key, sending updated $B K_{M_{9}}, B K_{V_{6}}$ and $B K_{V_{2}}$ to their corresponding trust sets $\left\{M_{5}, M_{6}, M_{7}, M_{9}\right\}$, $\left\{M_{5}, M_{6}, M_{7}, M_{9}\right\}$ and $\left\{M_{1}, M_{2}, M_{3}, M_{4}\right\}$ respectively.

Interval Multicast Protocol. $A F T D$ can also realize secure interval multicast, in which a group member wants to send data to a subgroup of group $G$. This problem is discussed by Gouda et al. [17], who describe a new use of key trees. They are concerned about using the existing subgroup keys in the key tree to securely multicast data to different subgroups within the group. Unlike their approach, which depends on a centralized key server to maintain the unique key tree and manage all keys, AFTD solves this problem in a distributed fashion. For the detailed algorithm, please refer to [12]. 
Updating Secret Keys \& Secret Shares. In AFTD, each group member is required to update its Diffie-Hellman keys before each group session, or during a session when it is selected as a sponsor on a member's leaving. Source authentication of the updated blinded keys is guaranteed by the sender's RSA signature. Further, to ensure the long-term secrecy of the RSA keys, AFTD requires each group member to renew its RSA key pair periodically, and send it to its trust set securely using its current RSA secret key. AFTD adopts the proactive secret share update algorithm in [18] to periodically update the system secret shares to invalidate compromised secret shares.

\subsection{Security of Trusted Authority}

The trusted authority, which may be distributed, is on-line during initialization, but remains offline subsequently. During initialization, the TA distributes valid key certificates and secret shares of its secret key $S K$, so that the function of key authentication can be realized and distributed across appropriate trust sets. Since the duration of initialization is relatively short, it is safe for us to use the $\mathrm{TA}$ at that time.

During the rekeying phase, the trusted authority may be approached by new group members for authentication and creation of valid key certificates for them. In this mode, the trusted authority works offline, in that it only communicates with new group members, making compromises of TA unlikely.

\subsection{The Number of Rekeying Messages Received}

On a rekeying event, all members in the trust sets of the nodes on the sponsor's key path will receive an updated blinded key. As in [1], we use the term co-path for the set of siblings of each node on the key path of member $M_{i}$. The nodes on a co-path have disjoint subtrees, so that the set of leaf nodes for these subtrees are also disjoint. Thus, the leaf nodes of each node in the co-path fall into clusters with minimal overlaps.

Because of the way they are defined(Section 4.2), the trust sets of the nodes on the key path of member $M_{i}$ have small overlap. Consequently, each group member receives nearly the same number of rekeying messages in our scheme. For example, in Figure 3, $V_{6}$ and $V_{2}$ are on the key path of the sponsor $M_{7} . V_{5}$ and $V_{1}$ are on the co-path of $M_{7}$, which have disjoint subtrees, so that $V_{6}$ an $V_{2}$ have disjoint trust sets. $V_{6}$ 's trust set is $\left\{M_{5}, M_{6}, M_{7}, M_{8}\right\}$, and $V_{2}$ 's trust set is $\left\{M_{1}, M_{2}, M_{3}, M_{4}\right\}$. Each member of these trust sets will receive one updated blinded key.

\section{Performance Analysis}

\subsection{Communication Overheads}

The Initialization Phase. The communication overhead is measured by the number of the messages. In the initialization phase of $A F T D$, the trusted authority distributes the certificates of each node in the key tree to its trust set. 
Since every node in each trust set receives a message, the overall communication overhead of the first phase is measured by the number of nodes in all trust sets.

If $n$ is the group size and $h_{V_{i}}$ is the height of $V_{i}$, there are at most $2^{\lg n-h_{V_{i}}}$ leaf nodes on the subtree of $V_{i}$ 's sibling node. Since the cluster size is $k$, the number of shares needed to reconstruct the certificate, these nodes will fall into at most $\left\lceil\frac{2^{\lg n-h_{V}}}{k}\right\rceil$ clusters. Hence, the size of $V_{i}$ 's trust set is no more than $\left\lceil\frac{2^{\lg n-h_{V_{i}}}}{k}\right\rceil \cdot k<2^{\lg n-1-h_{V_{i}}}+2 k$ nodes. As the maximum number of nodes in each level $h_{V_{i}}$ is $2^{h_{V_{i}}}$, the communication overhead of each level is at most $2^{h_{V_{i}}} \cdot\left(2^{\lg n-h_{V M_{i}}}+2 k\right)$. Therefore, we can compute the overall communication overhead in the initialization phase as $C_{\text {Initial }}=\sum_{i=1}^{\lg n} 2^{i} \cdot\left(2^{\lg n-i}+2 k\right)=$ $O(n \log n)$.

The Rekeying Phase. In the rekeying phase of AFTD, when a new member joins or an old member leaves, keys for nodes on the sponsor node's key path must be updated and multicast. Hence for each level of the key tree, only one node's blinded key has been updated and must be multicast to its trust set. Since the size of the trust set of the nodes on level $h_{V_{i}}$ is at most $\left\lceil\frac{2^{\lg n-h_{V_{i}}}}{k}\right\rceil \cdot k<$ $2^{\lg n-h_{V_{i}}}+2 k$ nodes, we can compute the overall communication in this stage as $C_{\text {Rekey }}=\sum_{i=1}^{\lg n}\left(2^{\lg n-i}+2 k\right)=O(n)$.

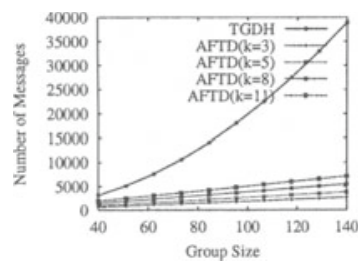

(a) Initialization Phase

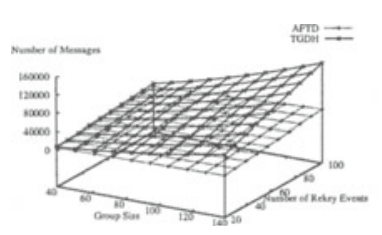

(d) Total Communication $\operatorname{Overhead}(k=11)$

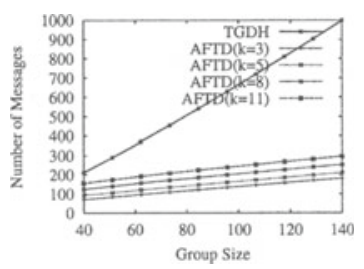

(b) Rekeying Phase

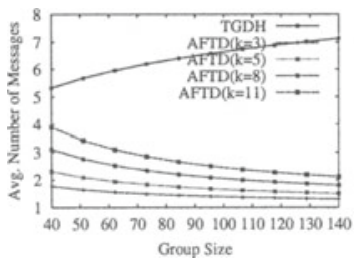

(e) Avg Number of Messages

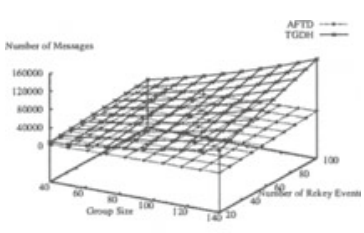

(c) Total Communication Overhead $(k=5)$

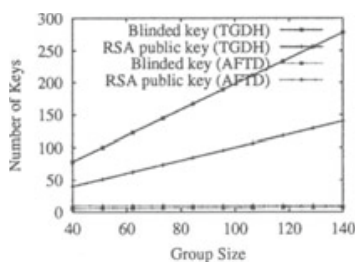

(f) Storage Requirement $(k=5)$

Fig. 5. Communication \& Storage Overheads 
Table 1. Performance of $T G D H$ and AFTD Compared

\begin{tabular}{|c|c|c|c|c|}
\hline & \multicolumn{2}{|c|}{ Communication Overhead } & \multicolumn{2}{c|}{ Storage Requirement } \\
\cline { 2 - 5 } & Initialization Phase & Rekeying Phase & Blinded Key & RSA Public Key \\
\hline$T G D H$ & $O\left(n^{2}\right)$ & $O(n \log n)$ & $O(n)$ & $n$ \\
\hline$A F T D$ & $O(n \log n)$ & $O(n)$ & $O(\log n)$ & $2 k$ \\
\hline
\end{tabular}

Figure 5(a) and (b) show the effects of different thresholds $k$ on the communication overheads of $A F T D$. They also compare the communication overhead of our scheme to that of $T G D H$. Figure 5(c) and (d) compare the total communication overhead of $A F T D$ with that of $T G D H$, where $k=5$ and 11 separately. Total communication overhead is defined as the combined communication overhead of initialization phase and multiple rekeying events. Clearly, the communication overhead of our scheme is significantly smaller than that of $T G D H$, especially ir. large dynamic group scenarios.

The value of $k$ is a system-dependent parameter, and represents a tradeoff between system security and fault-tolerance. Further, as seen from the above figures, the communication overhead of our scheme is insensitive to $k$.

Number of Messages Received on Rekeying. As explained in Section 4.5, each group member receives nearly the same number of rekeying messages in our scheme, because of the way trust sets are defined. Thus, the average number of messages on an rekeying event is a reasonable measure of communication overhead. Figure 5(e) compares the average number of messages received by each group member on an rekeying event. As the group size increases, the average number of messages received decreases to approximately one in our scheme, while it remains at $\lg n$ in $T G D H$. For example, when the group size is 128 , each group member receives around two rekeying message in our scheme, but about seven in TGDH. This demonstrates the scalability of AFTD in terms of the load experienced by group members.

\subsection{Computation Overhead and Storage Requirements}

Table 1 compares the performance of TGDH and AFTD. Due to space limitation, readers are referred [12] for a detailed analysis of computation and storage requirements.

\section{Conclusion}

In this paper, we have presented $A F T D$, an efficient, authenticated and faulttolerant tree-based key agreement protocol. Central to our technique is a threshold secret sharing based method to distribute the function of trusted authority to appropriate trust sets. Our performance analysis shows that our approach can significantly reduce the communication and storage overheads. 
Acknowledgement. This work is supported in part by grants from Tata Consultancy Services, Inc., and the Fault-Tolerant Networks program of Defense Advanced Research Projects Agency, under contract F30602-01-2-0536.

\section{References}

1. Kim, Y., Perrig, A., Tsudik, G.: Simple and fault-tolerant key agreement for dynamic collaborative groups. In: Proceedings of the CCS'00. (2000)

2. Steiner, M., Tsudik, G., Waidner, M.: Key agreement in dynamic peer groups. IEEE TRANSACTIONS on Parallel and Distributed Systems 11 (2000)

3. Perrig, A.: Efficient collabortive key management protocols for secure automonomous group communication. In: Proceedings of CrypTEC'99. (1999)

4. Wong, C., Gouda, M., Lam, S.: Secure group communication using key graphs. In: Proceedings of the ACM SIGCOMM'98, Vancouver, Canada (1998)

5. Wallner, D., Harder, E., Agee, R.: Key management for multicast: Issues and architecture. In: Internet Draft, draft-wallner-key-arch-01.txt. (1998)

6. Steiner, M., Tsudik, G., Waidner, M.: Cliques: A new approach to group key agreement. In: Proceedings of the ICDCS'98, Amsterdam, Netherlands (1998)

7. Ateniese, G., Steiner, M., Tsudik, G.: New multiparty authentication services and key agreement protocols. IEEE Journal of Selected Areas in Communications 18 (2000)

8. Pereira, O., Quisquater, J.: A security analysis of the cliques protocols suites. In: Proceedings of the 14-th IEEE Computer Security Foundations Workshop. (2001)

9. Lee, P., Lui, J., Yau, D.: Distributed collaborative key agreement protcols for dynamic peer groups. In: Proceedings of the ICNP'02. (2002)

10. Lee, P., Lui, J., Yau, D.: Distributed collaborative key agreement protcols for dynamic peer groups. Technical report, Dept. of Computer Science and Engineering, Chinese University of Hong Kong (2002)

11. Kong, J., Zerfos, P., Luo, H., Zhang, L.: Providing robust and ubiquitous security support for mobile ad-hoc networks. In: Proceedings of the ICNP'01. (2001)

12. Zhou, L., C.V.Ravishankar: Efficient, authenticated, and fault-tolerant key agreement for dynamic peer groups. Technical Report 88, Dept. of Computer Science and Engineering, University of California, Riverside (2003)

13. Narasimha, M., Tsudik, G., Yi, J.H.: On the utility of distributed cryptography in p2p and manets: the case of membership control. In: Proceeding of the ICNP'03. (2003)

14. Amir, Y., Kim, Y., Nita-Rotaru, C., Tsudik, G.: On the performance of group key agreement protocols. In: Proceedings of the ICDCS'02. (2002)

15. Amir, Y., Nita-Rotaru, C., Stanton, J., Tsudik, G.: Scaling secure group communication systems: Beyong peer-to-peer. In: Proceedings of the DISCEX'03, Washington DC (2003)

16. Shamir, A.: How to share a secret. Communications of the ACM 22 (1979)

17. M.G.Gouda, Huang, C., E.N.Elnozahy: Key trees and the security of interval multicast. In: Proceedings of the ICDCS'02, Vienna, Austria (2002)

18. Herzberg, A., Jarecki, S., Krawczyk, H., Yung, M.: Proactive secret sharing or: How to cope with perpetual leakage. extened abstract, IBM T.J. (1995) 